\title{
Pedestrian Modelling: A Comparative Study Using Agent-Based Cellular Automata
}

\author{
Nicole Ronald and Michael Kirley \\ The University of Melbourne \\ Parkville, Victoria 3010, Australia \\ \{naron, mkirley\}@csse.unimelb.edu.au
}

\begin{abstract}
In this paper, we examine pedestrian population dynamics using agent-based cellular automata models. Each pedestrian is treated as an agent, mapped onto a 2-dimensional grid. The behaviour of each agent is modelled as a sequence of specific choices reflecting different levels of autonomy. Simulations of bi-directional agent movement for four behaviours in different environments (corridors of different widths with permanent blocks such as walls) are conducted in order to identify outcomes of the behaviours and recommend a strategy. The results suggest that the "lookahead" behaviour, whilst similar to the "deterministic" behaviour, was strategically the best. Little difference was found between the "floor fields" and "random walk" behaviours.
\end{abstract}

\section{Introduction}

Creating simulations of pedestrian behaviour is difficult [1]. Walking behaviour is largely unconscious, less constrained and consequently less predictable than vehicle traffic. However, predictions of pedestrian behaviour are required for making decisions about the design of pedestrian facilities or creating management plans for pedestrian events.

Many approaches have been developed for modelling pedestrian behaviour at both macroscopic/aggregate levels and microscopic/disaggregate levels [2]. Agent-based cellular automata (CA) represent one class of model, which encapsulate fundamental movement rules at the microscopic level. These models have been used mainly for experimenting with detailed design of pedestrian areas by modelling operational (ie. stepping) decisions. The rules developed for models of pedestrian behaviour are refined with the macroscopic behaviour in mind (such as lane formation) and are not accurately based on real-life operational behaviour 3 .

In this paper, we investigate some common behavioural rules used in agentbased CA models of pedestrian behaviour and identify similarities between simple rulesets and more complex and 'realistic' rulesets. Each pedestrian is treated as an agent, mapped onto a 2-dimensional grid of a CA. Here, the behaviour of each agent is modelled as a sequence of specific choices reflecting different levels of autonomy. An important contribution of this work is the comparative analysis of alternative agent behaviours. We limit our investigations to: (a) 
bi-directional agent movement in different environments (corridors of different widths with permanent blocks such as walls), and (b) examining the effects of pedestrian density and resulting macroscopic properties of the model. In the next section, the simulation environment is outlined, followed by a description of the behaviours modelled. In section 3, experimental results are presented. We conclude with a summary of the paper and the implications of this work.

\section{Simulation Model Framework}

\subsection{Agent-Based CA approach}

The main components in the model are the environment (CA grid) and the pedestrians (agents). This segregation between environment and agents provides the necessary flexibility to model individual pedestrian behaviour independently, and subsequently complex interactions can be captured. The environment can have global properties or cell properties. The cell properties form a field over the environment - that is, the Moore-neighbourhood cells can have an effect on a cell. In this study, we are dealing in abstract time and space, however, we could assign real-world sizes to the cell dimensions and time steps based on current pedestrian design theory. Agents occupy cells in the CA grid. They attempt to move towards their destination by selecting an unoccupied neighbouring site. Agents require a direction (either up or down) and the developer can create properties as they like (such as speed, familiarity, current cell). These properties are manipulated when the agent moves.

The simulation model has been developed in Java using object-oriented principles. It is essentially a framework, which permits the developer to create and manipulate their own environment and pedestrian properties. As such, it is similar to the geosimulation framework developed for geographic automata systems [1].

\subsection{Modelling Pedestrian Behaviours}

Many rules have been developed for agent-based CA models of pedestrian behaviour. In this study, we focus on four behaviours to compare their performance. Is the simple model as good as the more 'realistic' model?

Random walk. The simplest behaviour is a random walk. This involves randomly selecting a neighbouring existing cell (ie. one that is not blocked or outside the environment). This behaviour was included as a "base" case to compare against.

Deterministic. The next behaviour uses a deterministic rule-based agent. This agent tries to move forward. If the cell is occupied or does not exist (ie. it is outside the environment or is blocked), it tries forward right or forward left. If neither of these cells exist, it tries immediate left or immediate right. 
Lookahead. This behaviour uses a "lookahead" procedure to determine whether to continue in the same lane or change lanes [4]. In short, the pedestrians look ahead a number of cells in their current lane and the lanes to their left and right. If there is someone ahead of them moving in the same direction, then the gap distance is set at the actual distance to that pedestrian. If there is someone ahead of them who is moving in the opposite direction, then the gap distance is set to half the actual distance. The pedestrian then chooses the lane with the maximal gap and ties between gap distance are broken randomly.

Floor fields. This behaviour [5] uses the concept of static and dynamic floor fields to guide the pedestrians. The static floor field consists of the distance to a specific activity or exit. The dynamic floor field consists of the number of pedestrians who have passed through the cell on the way to a specific activity of exit. Each agent has a parameter that dictates whether they follow the static floor field more (representing those who are familiar with the environment) or the dynamic floor field more (representing those unfamiliar with the environment who will follow other pedestrian going to the same destination).

This behaviour has also been developed by [6] and we partially adopted a variation from this work. In crowded situations, the agents are surrounded by other agents, therefore all cells returned a probability of 0 . This meant the agent did not attempt to move, leading to total gridlock.

The crux of the behaviour is shown in equation 1 which shows the calculation of the transition probability from the current cell $i$ to a neighbouring cell $j$. Contributions to the probability are the static floor field $S$, the dynamic floor field $D$, the pedestrian's preference for moving in that direction $M$, whether the cell is occupied or not $n$ (which is always 1 - unoccupied - in this model) and whether the cell exists or not $e$. The probabilities are normalised by the factor $N$.

$$
p_{i j}=N M_{i j} D_{i j} S_{i j} n_{j} e_{j}
$$

In each time step, each agent chooses their next cell. If two or more agents have chosen the same cell, the collision rules decide who moves to that cell and who stays in their current cell. The rules are based on the principles in [7, however for this set of results the friction parameter was set to 0 .

\section{Experiments and Discussion}

\subsection{Model Parameters}

Two different environment sizes were used in this study. The environment was 200 cells long $(L)$ and the width was either 15 or 20 cells $(W)$. For some runs, a permanent blockage was introduced in the form of two doors at $L / 2$. Four density values $(D)$ were used to vary to the volume of agents $(V): D=\{0.1,0.3,0.5,0.7\}$ and $V=D \times W \times L$. These densities provide conditions ranging from free-flowing to very congested. Half of the agents started at the top of the environment and half at the bottom. The agents were randomly assigned entry times between 0 and $L / 2$ and a starting cell in the first row. 


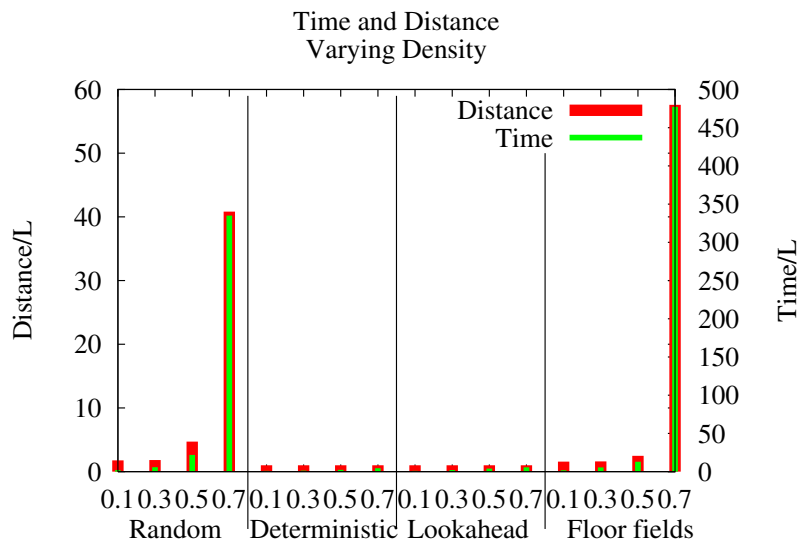

Fig. 1. Time and distance travelled divided by the length $L$ of the environment

Movement and collision resolution was synchronous and all agents moved at most one cell per timestep. A simulation trial was run until all pedestrians reached their destination or $L \times 100$, whichever occurred first. Each scenario (environment-agent behaviour) was run several times with different random seeds.

\subsection{Results}

A range of simulation model outputs were recorded during each trial, including the distance covered by each agent, the time they were in the model, the number of collisions, and entropy. All results presented are an average over all trials.

The time and distance plots (Figure 1) show that the agents using the random and floor fields behaviours take longer to move through the environment. The deterministic and lookahead behaviours are more efficient.

The stops due to collisions plots (Figure22) show that the agents using the random and floor fields behaviours are delayed due to collisions a similar amount. The deterministic behaviour performs well at low densities. However, this degrades quickly at higher densities. The lookahead behaviour appears to be approaching a maximum.

The snapshots (Figure 3) show the differences between the behaviours at the same point in time. The random and floor field behaviours cluster in the centre, whilst the deterministic agents have all moved to the right and out of each other's way. Given their rule set, which is to move right before left, this is expected. The lookahead behaviour is also clustered, but in one large cluster. The differences between densities for the same behaviour (lookahead) are also clear. At the time for the lowest density, the agents have almost reached their destinations. At the higher densities, a cluster has formed, but agents are still moving.

In order to investigate the population dynamics in more detail, the Shannon entropy $[8$ of agent movement throughout a trial were recorded. This was used to compare the similarity of behaviours. An inspection of the entropy plots (Figures 


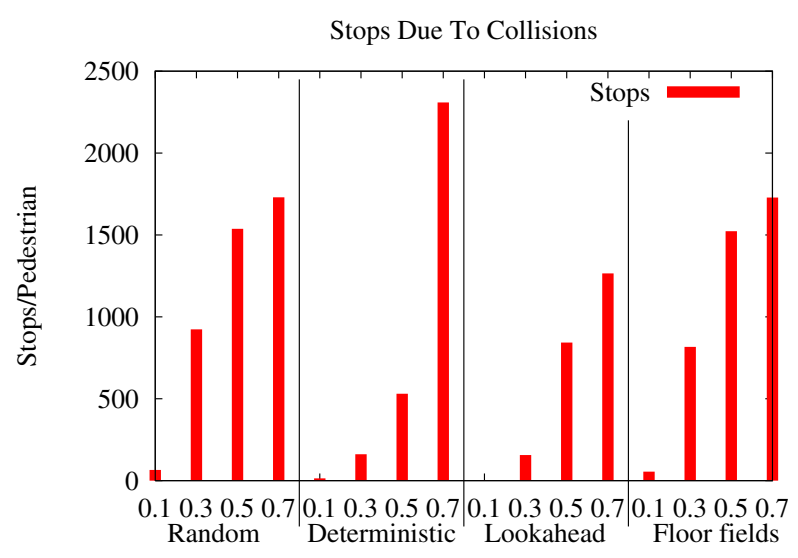

Fig. 2. The average number of times a pedestrian stops due to a collision

4 and 5) reveals that the peaks of the plots occur when the types of movement are evenly split ie. half the agents are moving and half are stopped. The troughs correspond to the situation where the agents are mostly doing the same thing ie. all moving or all stopped. The results for deterministic behaviour and the lookahead behaviour are similar, while the random and the floor fields behaviours are similar to each other. In this simple environment, the floor field behaviour is essentially an 'intelligent' random choice. The entropy plots also show that the behaviours go through similar phases throughout their run. Model runs with a larger number of pedestrians have similar peaks and troughs, but translated along the time axis.

For densities $30 \%$ and above, the deterministic and lookahead behaviours have a trough around $t=100$ and a peak around $t=150$ (Figure 4). At $t=100$, the agents who entered the model first have reached the centre. Up until this point, they could move freely as they were surrounded by pedestrians moving in their direction. However, at the centre they encounter pedestrians moving in the other direction and therefore collisions start occurring. At $t=150$, the two middle quarters are full of pedestrians, with very few pedestrians in the end quarters. The agents who entered the model first are encountering the last of the agents heading in the other direction. After this time, agents start to move freely again.

For the random and floor fields behaviours, there is a peak at $t=250$. As these behaviours are more likely to move sideways than the other behaviours, they take longer to reach the centre of the environment.

There are other peaks later on for some model runs. This usually occurs when a crowd has formed that has impeded a lot of pedestrians, however pedestrians are slowly making their way through. The peak occurs when there are more pedestrians free of the crowd and making their way to the exit than in the crowd still. The slight increase in width had minimal effect on the behaviour.

From the simulation results, it is clear that in this simple rectangular environment the floor field behaviour provides little advantage over the random behaviour. The floor field behaviour is more suited to a more complex situation 

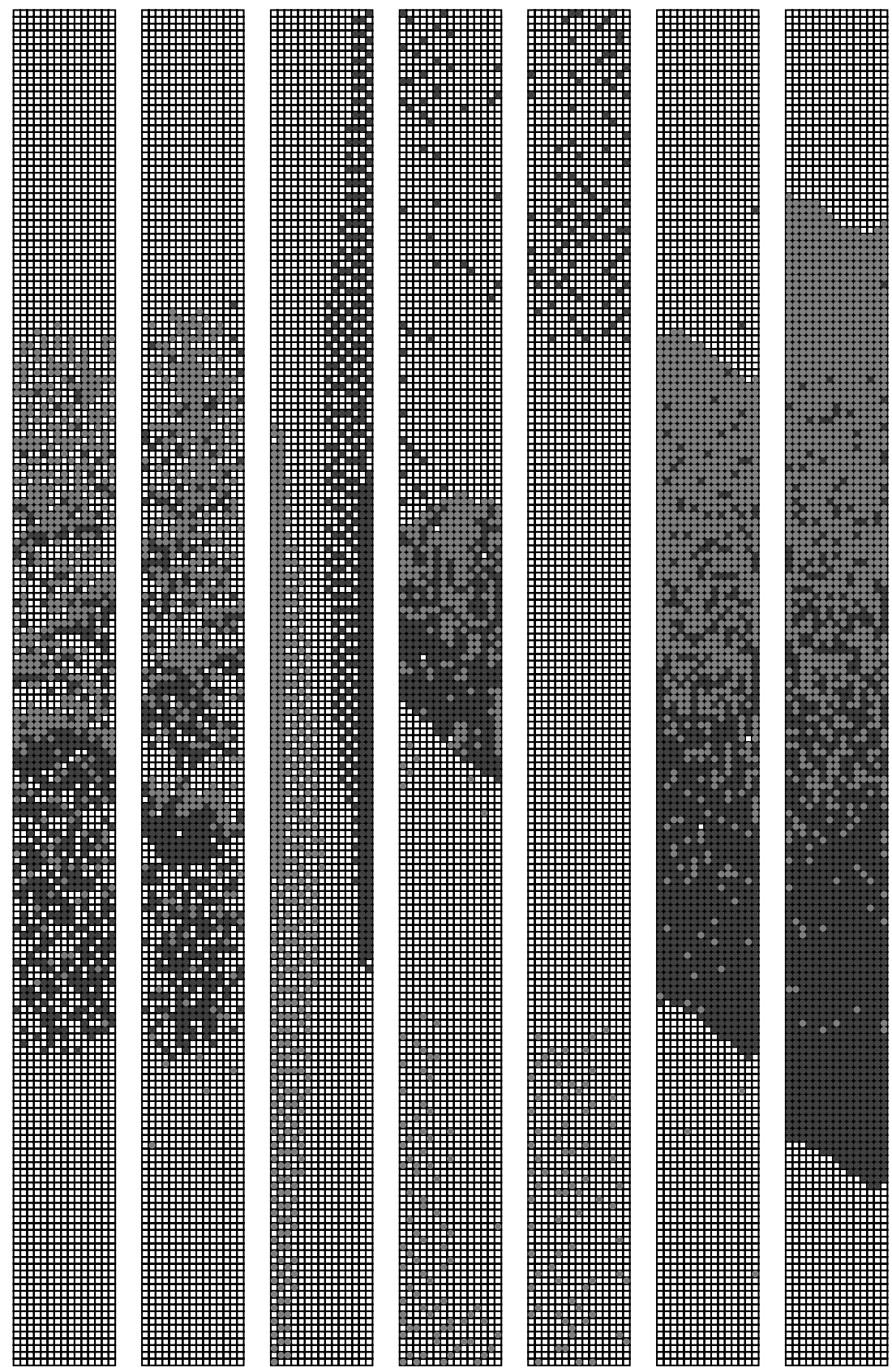

Fig. 3. Modelled crowds at the same time $(t=250)$ for different behaviour and densities. The dark grey pedestrians are moving upwards, the light grey downwards. From left: random-30\%, floor fields-30\%, deterministic-30\%, lookahead-30\%, lookahead-10\%, lookahead-50\%, lookahead- $70 \%$. 


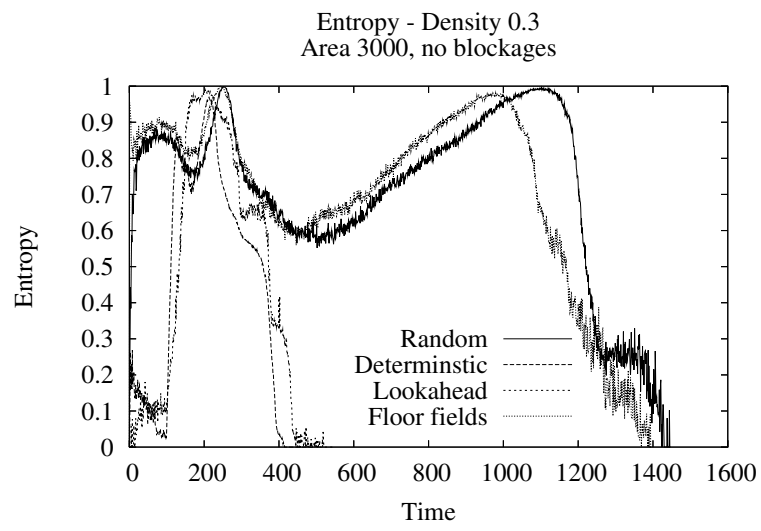

Fig. 4. Entropy plots for different behaviours with density $30 \%$

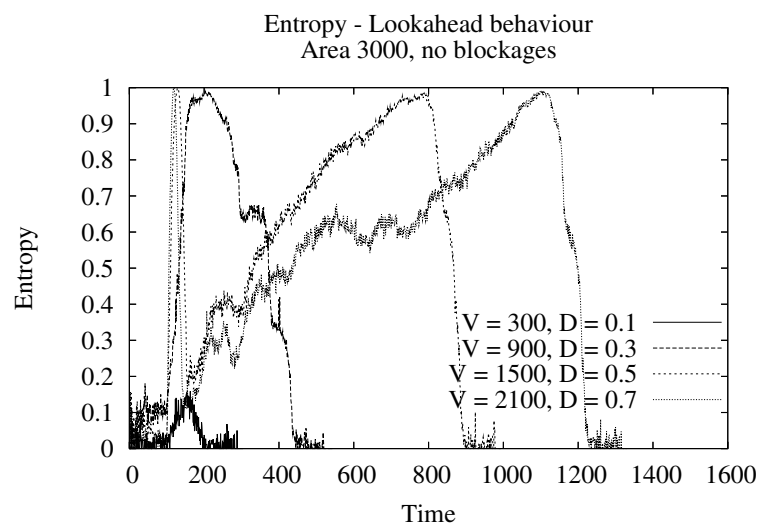

Fig. 5. Entropy plots for the lookahead behaviour at different densities

with many choices for exits and/or activities. The deterministic and lookahead behaviours were similar, however the lookahead behaviour performed better for collisions at higher densities and the differences between distance and time travelled were negligible.

\section{Conclusion}

The main contribution of this paper was the presentation of a comparative analysis of agent-based CA models of pedestrian behaviour of varying complexity.

Simulation experiments using different agent behaviours and environments (bidirectional corridors with varying traffic volume) suggest that the lookahead behaviour is the best strategy when moving through a simple environment, in terms of the travel time and delays. The deterministic behaviour is also a good strategy. The floor fields behaviour was the most complicated and 'realistic' 
behaviour, however the random and floor field behaviours do not perform as well in simple environments. Similarities were identified in the outputs between lookahead and deterministic and between random and floor fields, by investigating the entropy plots.

In future work, we aim to increase the complexity of the model (both the environment and range of agent behaviours). The model will be populated with agents exhibiting heterogeneous behaviours. Variable speed can also be introduced to the model. Assigning real-world values to the cell sizes and speeds and validation (obviously at a smaller scale) will also be investigated.

\section{References}

1. Benenson, I., Torrens, P.N.: Geosimulation : automata-based modelling of urban phenomena. John Wiley \& Sons, Hoboken, NJ, USA (2004)

2. Harney, D.: Pedestrian modelling: current methods and future directions. Road \& Transport Research 11 (2002) 2-12

3. Hoogendoorn, S., Bovy, P.H.L.: Normative pedestrian behaviour theory and modelling. In Taylor, M.A.P., ed.: Proceedings of the 15th International Symposium on Transportation and Traffic Theory. (2002)

4. Blue, V.J., Adler, J.L.: Cellular automata microsimulation of bi-directional pedestrian flows. Transportation Research Record 1678 (2000) 125-141

5. Schadschneider, A.: Cellular automaton approach to pedestrian dynamics - theory. In Schreckenberg, M., Sharma, S., eds.: Pedestrian and Evacuation Dynamics. Springer-Verlag, Berlin (2001)

6. Henein, C.M., White, T.: Agent-based modelling of forces in crowds. In Davidsson, P., Gasser, L., Logan, B., Takadama, K., eds.: Multi-Agent and Multi-Agent-Based Simulation. Volume 3415 of Lecture Notes in Computer Science. Springer-Verlag (2004) 173-184

7. Kirchner, A., Nishinari, K., Schadschneider, A.: Friction effects and clogging in a cellular automaton model for pedestrian dynamics. Physical Review E 67 (2003)

8. Boschetti, F., Prokopenko, M., Macreadie, I., Grisogono, A.M.: Defining and detecting emergence in complex networks. In Khosla, R., Howlett, R.J., Jain, L.C., eds.: Knowledge-Based Intelligent Information and Engineering Systems: 9th International Conference, KES 2005. Volume 3684 of Lecture Notes in Computer Science. Springer-Verlag (2005) 573-580 